\title{
Atypical Presentation of Myocardial Infarction in a Young Patient With Polycystic Ovarian Syndrome
}

\author{
Shobha Mandal ${ }^{1}$, Ravi R. Pradhan ${ }^{2}$, Barbara Mols Kowalczewski ${ }^{3}$ \\ 1. Internal Medicine, Guthrie Robert Packer Hospital, Sayre, USA 2. Internal Medicine, Tribhuvan University Institute \\ of Medicine, Kathmandu, NPL 3. Endocrinology, Guthrie Robert Packer Hospital, Sayre, USA
}

Corresponding author: Shobha Mandal, shobha.mandal@guthrie.org

\begin{abstract}
Polycystic ovarian syndrome (PCOS) is one of the common endocrinopathy noted in women of childbearing age groups. Patients with PCOS have increased cardiovascular risk factors compared with age-matched control; hence, these patients are believed to be at an increased risk for cardiovascular events. Here, we report a case of a young female with PCOS, who presented with atypical back pain in the thoracic region. Initially, her electrocardiogram (EKG) and troponin were normal; hence, it was thought to be a muscle spasm but the back pain continued; repeat EKG and troponin came abnormal suggestive of myocardial infarction (MI). The patient underwent primary percutaneous coronary intervention and was discharged on dual antiplatelet therapy. MI is common in patients with PCOS. MI is the most important differential diagnosis in any patients of PCOS presenting with chest pain or back pain. Early diagnosis and prompt treatment of MI in patients with PCOS prevent adverse outcomes.
\end{abstract}

Received 07/08/2020 Review began 07/13/2020 Review ended 07/15/2020 Published 07/31/2020

๑) Copyright 2020 Mandal et al. This is an open access article distributed under the terms of the Creative Commons Attribution License CC-BY 4.0., which permits unrestricted use, distribution, and reproduction in any medium, provided the original author and source are credited.
Categories: Cardiology, Endocrinology/Diabetes/Metabolism, Internal Medicine

Keywords: polycystic ovarian syndrome, pcos, young female, atypical back pain, myocardial infarction, primary percutaneous coronary intervention

\section{Introduction}

Polycystic ovarian syndrome (PCOS) is one of the common endocrinopathy noted in women of childbearing age groups, with a prevalence ranging from $4 \%$ to $12 \%$ globally [1]. Patients with PCOS have insulin resistance, dyslipidemia, obesity, androgen excess, and infertility [2-4]. All these are risk factors for cardiovascular diseases. Because of association with multiple metabolic risk factors in patients with PCOS at a young age, there is an increased chance of developing cardiovascular diseases in this group of patients compared to their age-matched controls [5]. However, evidence generated so far is inconclusive for the risk of MI, and cardiovascular risk profiles may vary with PCOS phenotype, age, ethnicity, and body mass index (BMI). A prospective study conducted by Dahlgren et al. in 1992 showed that the risk of myocardial infarction (MI) was seven times more in peri- and postmenopausal women with PCOS compared to healthy controls [4]. Here, we present a case of a young woman with PCOS presenting with atypical back pain in the thoracic region, which was later diagnosed as MI.

\section{Case Presentation}

A 36-year-old, Caucasian female, nonsmoker, with a past medical history of PCOS, anxiety, and depression came to the ED with a complaint of back pain in the thoracic region for 10 days. The pain started suddenly after she lifted a typewriter weighing 60 pounds. The pain was throbbing in nature, 5/10 in intensity, radiating to both arms, worsened with any movement, and lasted for several hours. She was taking over-thecounter tylenol with minimal improvement in the pain. She came to the ED as the pain persisted. On arrival, she rated her pain as severe. She was vitally stable and general physical exam was benign. Routine laboratory workup and troponin were within normal limits. She was suspected to have muscle spasm of the back and was treated with stat doses of ketorolac $30 \mathrm{mg}$ intramuscularly, cyclobenzaprine $10 \mathrm{mg}$ orally as well as a lidocaine transdermal patch. She reported improvement in her back pain and was discharged on cyclobenzaprine $10 \mathrm{mg}$ orally three times a day for seven days. After reaching home she started having the back pain again without any improvement with muscle relaxant (cyclobenzaprine). She also reported having a new onset of nausea and one episode of vomiting. However, she did not have a fever, cough, hemoptysis, and shortness of breath. She came to the ED for further evaluation. On detailed discussion, she reported that her father and grandfather had MI in their fifties, and mother died at the age of 24 during childbirth due to unknown reasons.

On physical examination, the patient appeared anxious. The temperature was $36.7^{\circ} \mathrm{C}$ (normal $=36.5^{\circ} \mathrm{C}$ $37.3^{\circ} \mathrm{C}$ ), pulse 121 beats per minute (normal $=60-100$ beats per minute), blood pressure $140 / 93 \mathrm{mmHg}($ normal < 120/80 $\mathrm{mmHg}$ ), respiratory rate 20 breaths per minute (normal = 12-18 breaths per minute), and oxygen saturation $96 \%$ (normal $=95 \%-100 \%$ ) while she was breathing ambient air. The body mass index (BMI) was $34.6 \mathrm{~kg} / \mathrm{m}^{2}$ (weight: $96.4 \mathrm{~kg}$, height: $1.67 \mathrm{~m}$ ). There was mild tenderness in the thoracic spine at 


\section{Cureus}

the T1-T3 level. The remainder of the physical examination was unchanged. Differential diagnosis included musculoskeletal pain, infectious process (spinal epidural abscess/pericarditis), aortic dissection, pulmonary embolism, and MI.

The details of lab work investigations are shown in Table 1.

\begin{tabular}{|c|c|c|c|}
\hline Parameters & Reference range, adults & Day 1 & Day 2 \\
\hline Platelets (per mm³) & $182000-369000$ & 358000 & 351000 \\
\hline White cell count (per $\mathrm{mm}^{3}$ ) & $4000-11000$ & 15710 & 17240 \\
\hline Neutrophils (\%) & $34.0-71.1$ & 76.9 & 78.6 \\
\hline Lymphocytes (\%) & $19.3-51.7$ & 13.4 & 12.4 \\
\hline Monocytes (\%) & 4.7-12.5 & 8.2 & 8.1 \\
\hline Eosinophils (\%) & $0.7-5.8$ & 0.7 & 0.1 \\
\hline Hemoglobin (g/dL) & $11.2-15.7$ & 14.7 & 12.9 \\
\hline Troponin I (ng/ml) & $0.000-0.034$ & 1.43 & 10.6 \\
\hline $\mathrm{N}$-terminal pro BNP (pg/ml) & $<125$ & 1,170 & NA \\
\hline Magnesium (mg/dL) & $1.6-2.3$ & 1.9 & 1.8 \\
\hline Lactic acid (mmol/L) & $0.7-2.1$ & 1.7 & NA \\
\hline Random blood sugar (mg/dL) & $<140$ & 121 & NA \\
\hline Creatinine (mg/dL) & $0.7-1.2$ & 0.8 & 0.8 \\
\hline Potassium (mmol/L) & $3.5-5.1$ & 4 & 4.3 \\
\hline Sodium (mmol/L) & $134-145$ & 140 & 139 \\
\hline CPK (U/L) & 30-135 & 373 & NA \\
\hline INR & $0.8-1.1$ & 0.97 & NA \\
\hline TSH (mlU/L) & $0.47-4.68$ & 1.33 & NA \\
\hline C-reactive protein (mg/L) & $<3$ & 0.7 & NA \\
\hline Cholesterol (mg/dL) & $<200$ & 225 & NA \\
\hline Triglycerides (mg/dL) & $<150$ & 145 & NA \\
\hline $\mathrm{HDL}(\mathrm{mg} / \mathrm{dL})$ & $40-59$ & 33 & NA \\
\hline LDL (mg/dL) & $100-129$ & 164 & NA \\
\hline
\end{tabular}

\section{TABLE 1: Laboratory parameters of the patient on day 1 and day 2.}

NA: Not available; BNP: Brain natriuretic peptide; CPK: Creatine phosphokinase; INR: International normalization ratio; TSH: Thyroid stimulating hormone; HDL: High density lipoprotein, LDL: Low density lipoprotein

Blood culture was negative for any growth. Urine drug screening for amphetamines, barbiturates, benzodiazepines, cannabinoids, cocaine, methadone, opiates, oxycodone, phencyclidine, and propoxyphene was negative.

CT angiography of the chest, abdomen, and pelvis was negative for pulmonary embolism, aortic aneurysm or dissection, and contrast-enhanced CT scan of the thoracic spine was negative for epidural abscess, fracture, or malalignment. Electrocardiogram (EKG) was initially normal (Figure 1). 


\section{Cureus}

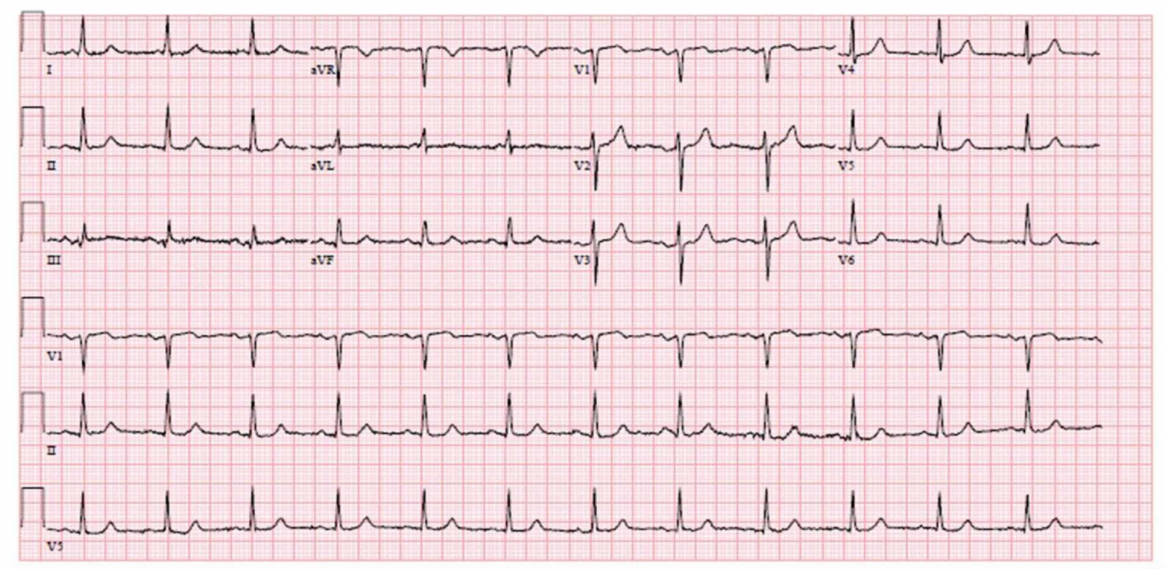

FIGURE 1: EKG on day 1 with normal findings.

EKG, electrocardiogram

Repeat EKG the next day showed sinus tachycardia, and Q-wave in lead III, and V1-V3 (Figure 2).

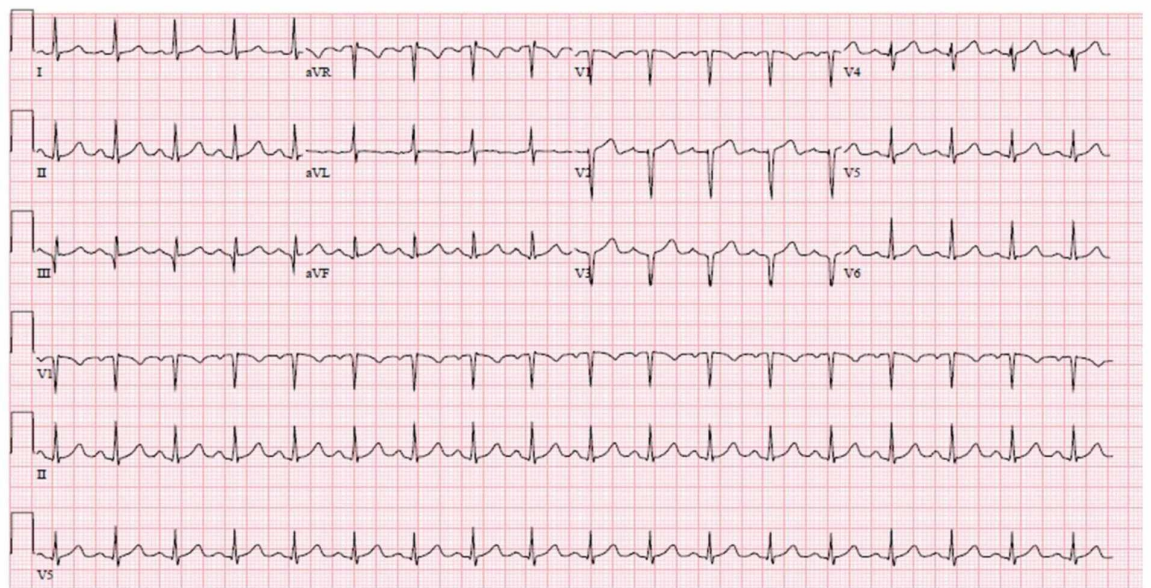

FIGURE 2: EKG (second day) showing sinus tachycardia, and Q-waves in lead III, and V1 to V3.

EKG, electrocardiogram

Echocardiogram showed hypokinesis of the left ventricular mid to apical, anterior septum, apical cap, and inferior apical wall.

A provisional diagnosis of acute MI was formulated based on ongoing chest pain, dynamic EKG changes, rising troponin I on serial monitoring, and echocardiographic findings. The patient was started on a heparin drip and was medically managed with aspirin $325 \mathrm{mg}$ orally, metoprolol $25 \mathrm{mg}$ orally, and sublingual nitroglycerin $0.4 \mathrm{mg}$. The patient was transferred immediately to the cardiac catheterization lab for left heart catheterization with angiography. Coronary angiography showed 99\% ostial left anterior descending (LAD) artery stenosis, mild luminal irregularities in the left circumflex artery, and right coronary artery (RCA) dominance. There was no stenosis noted in the left main artery and RCA (Figure 3). 


\section{Cureus}

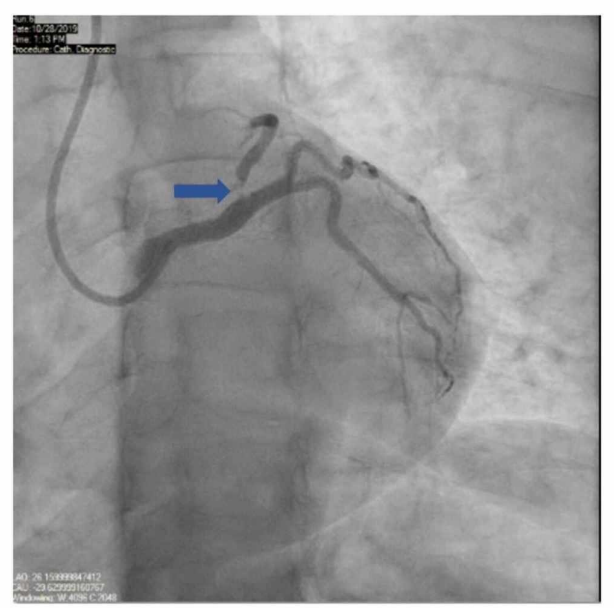

\section{FIGURE 3: Pre-intervention coronary angiography.}

Coronary angiography showed $99 \%$ ostial LAD artery stenosis, mild luminal irregularities in the left circumflex artery, and RCA dominance. The blue arrow points to the location of stenosis

LAD, left anterior descending; RCA, right coronary artery

A drug eluting stent (DES) was placed to the ostial LAD artery without any complications (Figure 4).

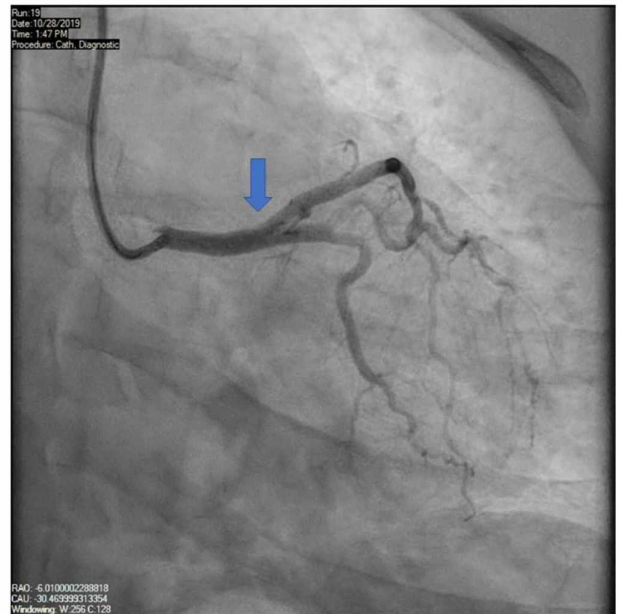

\section{FIGURE 4: Post-intervention angiography.}

The blue arrow points to restoration of blood flow after drug eluting stent placement in the ostial LAD artery

LAD, left anterior descending

She was discharged on oral medicines (aspirin $81 \mathrm{mg}$ daily, atorvastatin $80 \mathrm{mg}$ daily, metoprolol $50 \mathrm{mg}$ BID, nitroglycerine $0.4 \mathrm{mg}$ sublingual as needed, and prasugrel $10 \mathrm{mg}$ daily) for secondary prevention. Dual antiplatelet therapy (aspirin and prasugrel) was continued for one year.

\section{Discussion}

Polycystic ovarian syndrome is one of the most common endocrine problems in women of childbearing group with a prevalence ranging from $4 \%$ to $12 \%$ globally [1-3]. PCOS can be diagnosed based on four key diagnostic features (ovulatory dysfunction, hirsutism, hyperandrogenism, and multiple ovarian cysts on ultrasound) $[1,6]$. It is the most frequent cause of anovulatory infertility, hirsutism, metabolic abnormalities, and also increases long-term risk for development of type 2 diabetes mellitus, endometrial 
Myocardial infarction is the leading cause of death in women worldwide [3]. Postmenopausal women were affected the most, but it is also found to be increasing in younger women [7]. In younger women, causes of acute MI include arterial plaque, microvascular dysfunction, vasospasm, or spontaneous coronary artery dissection [3]. PCOS has an increased risk of coronary artery disease due to chronic inflammation, oxidative stress, impaired fibrinolysis, endothelial dysfunction, increased intimal thickness, increased coronary artery calcification, and impaired pulse wave velocity [8].

The association of PCOS with metabolic syndrome (including increased androgen, estrogen, homocysteine level, insulin) further increases the risk of CAD [2-4, 7, 9-10]. Both obese and nonobese patients with PCOS are found to be at increased risk [11]. Oral contraceptive pills further increase the risk of thromboembolic events and MI as it alters almost all the homeostatic parameters including plasma level of coagulation factors, anticoagulants proteins, and proteins involved in fibrinolytic pathways [10]. Due to the association with multiple risk factors, studies have found two-fold increased risk of CAD in patients with PCOS compared to women without PCOS [5].

As PCOS is associated with a range of reproductive, obstetric, metabolic and psychosocial features, management should focus on multiple aspects like metabolic, reproductive, and psychosocial features [3, 8]. The patient should also be monitored and managed for long-term metabolic complications [11-12].

\section{Conclusions}

Polycystic ovarian syndrome is a common and increasingly challenging endocrine problem in women of childbearing age. Despite the association of PCOS with multiple comorbidities, this medical condition is not treated properly due to a lack of awareness and treatment guidelines. Patients with PCOS are found to be at increased risk for MI, stroke, and aortic dissection. The purpose of our case report is to raise awareness regarding the association of PCOS with MI, and our focus as a physician should provide emotional and psychosocial support to the patient. In conclusion, this case suggests an association of PCOS with MI. Further studies are needed to decrease the risk of cardiovascular disease in PCOS patients. Proper evidencebased guidelines are needed for appropriate prevention and management to reduce morbidity and mortality associated with it.

\section{Additional Information \\ Disclosures}

Human subjects: Consent was obtained by all participants in this study. Conflicts of interest: In compliance with the ICMJE uniform disclosure form, all authors declare the following: Payment/services info: All authors have declared that no financial support was received from any organization for the submitted work. Financial relationships: All authors have declared that they have no financial relationships at present or within the previous three years with any organizations that might have an interest in the submitted work. Other relationships: This case abstract was accepted for ENDO 2020 and got published in Journal of Endocrine Society. J Endocr Soc. 2020 May 8; 4(Suppl 1): SUN-LB1. Published online 2020 May 8. doi: 10.1210/jendso/bvaa046.2004.

\section{References}

1. Azziz R, Carmina E, Dewailly D, et al.: Positions statement: criteria for defining polycystic ovary syndrome as a predominantly hyperandrogenic syndrome: an Androgen Excess Society guideline. J Clin Endocrinol Metab. 2006, 91:4237-4245. 10.1210/jc.2006-0178

2. Broekmans FJ, Knauff EA, Valkenburg O, et al.: PCOS according to the Rotterdam consensus criteria: change in prevalence among WHO-II anovulation and association with metabolic factors. BJOG. 2006, 113:1210-1217. 10.1111/j.1471-0528.2006.01008.x

3. Chandrasekhar J, Gill A, Mehran R: Acute myocardial infarction in young women: current perspectives . Int J Womens Health. 2018, 10:267-284. 10.2147/IJWH.S107371

4. Dahlgren E, Janson PO, Johansson S, et al.: Polycystic ovary syndrome and risk for myocardial infarction. Evaluated from a risk factor model based on a prospective population study of women. Acta Obstet Gynecol Scand. 1992, 71:599-604. 10.3109/00016349209006227

5. de Groot PC, Dekkers OM, Romijn JA, et al.: PCOS, coronary heart disease, stroke and the influence of obesity: a systematic review and meta-analysis. Hum Reprod Update. 2011, 17:495-500. 10.1093/humupd/dmr001

6. Dunaif A, Graf M, Mandeli J, et al.: Characterization of groups of hyperandrogenic women with acanthosis nigricans, impaired glucose tolerance, and/or hyperinsulinemia. J Clin Endocrinol Metab. 1987, 65:499-507. 10.1210/jcem-65-3-499

7. Hull MG: Epidemiology of infertility and polycystic ovarian disease: endocrinological and demographic studies. Gynecol Endocrinol. 1987, 1:235-245. 10.3109/09513598709023610

8. Moran L, Teede H: Metabolic features of the reproductive phenotypes of polycystic ovary syndrome . Hum Reprod Update. 2009, 15:477-488. 10.1093/humupd/dmp008

9. Saadeh N, Alfaqih MA, Mansour H, et al.: Serum homocysteine is associated with polycystic ovarian syndrome in Jordan. Biomed Rep. 2018, 9:439-445. 10.3892/br.2018.1149 


\section{Cureus}

10. Tchaikovski SN, Rosing J: Mechanisms of estrogen-induced venous thromboembolism. Thromb Res. 2010, 126:5-11. 10.1016/j.thromres.2010.01.045

11. Teede H, Deeks A, Moran L: Polycystic ovary syndrome: a complex condition with psychological,

reproductive and metabolic manifestations that impacts on health across the lifespan. BMC Med. 2010, 8:41. 10.1186/1741-7015-8-41

12. Zhao L, Zhu Z, Lou H, et al.: Polycystic ovary syndrome (PCOS) and the risk of coronary heart disease (CHD): a meta-analysis. Oncotarget. 2016, 7:33715-33721. 10.18632/oncotarget.9553 